\title{
The inner $\mathbf{3 0 0}$ parsecs of the Milky Way seen by H.E.S.S.: a Pevatron in the Galactic Centre
}

\author{
Emmanuel Moulin ${ }^{1, a}$ for the H.E.S.S. collaboration \\ ${ }^{1}$ DRF/Irfu, Service de Physique des Particules, CEA Saclay, F-91191 Gif-Sur-Yvette Cedex, France
}

\begin{abstract}
The Galactic Centre region has been observed by the High Energy Stereoscopic System (H.E.S.S.) array of ground-based Cherenkov telescopes since 2004 leading to the detection of the very-high-energy $\gamma$-ray source HESS J1745-290 spatially coincident with the supermassive black hole Sagittarius A*. Diffuse TeV gamma-ray emission has been detected along the Galactic plane, most likely due to hadronic cosmic-ray interactions with the dense gas of the Central Molecular Zone. The rich 2004-2013 dataset permits detailed spectral and morphological studies of the diffuse emission in the inner $300 \mathrm{pc}$ of the Galactic Centre region. The new results provide an important statement regarding the location and origin of the accelerator of $\mathrm{PeV}$ protons. The H.E.S.S. observations of the Pevatron are discussed in the context of the origin of Galactic cosmic rays.
\end{abstract}

\section{Introduction}

The Galactic center (GC) region has been longtime observed from radio to X-ray wavelengths with increasing accuracy. Since more than a decade with the advent of the LAT instrument onboard the Fermi satellite and ground-based Cherenkov telescopes, observations of the inner GC region provide a efficient probe of the most violent phenomena in the universe. The very-high-energy (VHE, $\mathrm{E}_{\gamma}>100$ $\mathrm{GeV}) \gamma$-ray observations with arrays of imaging atmospheric Cherenkov telescopes (IACT) provide crucial insights to study cosmic-ray (CR) particle acceleration and propagation up to PeV energies.

While totally obscured at optical wavelengths, the GC is visible in a broad-band spectrum of electromagnetic wavelengths. The infrared observations probe star formation regions where the UV/optical light from massive young stars is converted by dust to infrared light. Non-thermal radio, $\mathrm{X}$-ray and $\gamma$-ray radiations probe charged particles accelerated to high energies in cosmic accelerators such a supernova remnants, pulsar wind nebulae, or massive star forming regions. Radio and X-ray emissions result from energetic electron interaction with magnetic fields through synchrotron radiation while $\mathrm{GeV} / \mathrm{TeV}$ emissions are produced by bremsstrahlung and inverse Compton of electrons scattering off gas or ambient radiation fields. Another mechanism of high-energy ( $\mathrm{HE}, 10 \mathrm{MeV}$ $100 \mathrm{GeV}$ ) and VHE $\gamma$-ray emissions proceeds through $\pi_{0}$ decays produced by inelastic collision of energetic protons and nuclei with the ambient gas.

During the last decade, detailed studies of the HE/VHE observations of the GC shed light to acceleration mechanisms taking place in this region. Observations with the Fermi-LAT instrument

\footnotetext{
a e-mail: emmanuel.moulin@cea.fr
} 
and IACTs such as H.E.S.S., MAGIC and VERITAS, led to the detection of numerous sources in the $\mathrm{HE} / \mathrm{VHE}$ regimes such as the pulsar wind nebula G0.9+0.1, supernova remnants, a central emission coincident in position with the supermassive black hole Sagittarius (Sgr) A*, as well as a diffuse $\mathrm{GeV} / \mathrm{TeV}$ emission.

\section{A strong TeV emission from the Galactic Center}

The GC has been long time recognized as a multi-TeV particle accelerator and then been subject to IACT observations of utmost importance. A central VHE signal has been detected in 2004 by H.E.S.S. [1], hereafter referred as to HESS J1745-290, and further observations with H.E.S.S. led to the detection an energy cut-off in the $10 \mathrm{TeV}$ energy range [2]. With 10 years of observations, H.E.S.S. provides the most detailed view so far at VHE (see Fig. 1). The position of the HESS
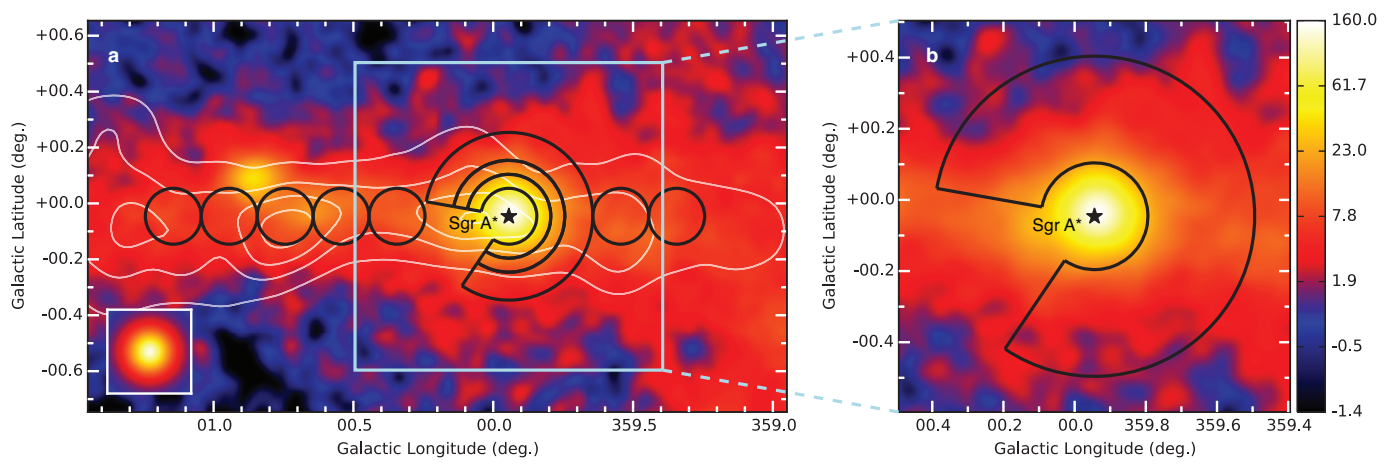

Figure 1. VHE $\gamma$-ray image of the Galactic Centre region. Left panel: The black lines outline the regions used to calculate the cosmic-ray energy density throughout the central molecular zone. A section of $66^{\circ}$ is excluded from the annuli to avoid contamination from a newly detected source. White contour lines indicate the density distribution of molecular gas, as traced by its CS line emission. The location of Sgr A* is given by the black star. The inset shows the simulation of a point-like source. Right panel: Zoomed view of the inner $70 \mathrm{pc}$ and the contour of the region used to extract the spectrum of the diffuse emission.

J1745-290 centroid is coincident with Sgr A* and the pulsar wind nebula G359.95-0.04 positions within 13" [3]. The energy spectrum of HESS J1745-290 derived from 10 year observations with H.E.S.S. is shown in the left panel of Fig. 2. Despite the rich statistics accumulated so far towards the GC, the nature of HESS J1745-290 is still unknown and compelling production mechanisms for this VHE emission possibly include: $(i)$ a CR source accelerating high energy protons in the vicinity of Sgr A* which produces VHE $\gamma$-rays from $\pi_{0}$ decays originating from hadronic CR penetrating the interstellar medium gas; (ii) the pulsar wind nebula G359.95-0.04; and (iii) a spike of annihilating dark matter particles. With the detection of the 2FHL J1745.7-2900 and 3FGL J1745.6-2859c sources with Fermi-LAT, the GeV emission from the pulsar wind nebula model significantly underestimates by far the luminosity of these sources, given the pronounced peak-like structure exhibited by the inverse Compton emission. Assuming the GeV source 3FGL J1745.6-2859c and the TeV source HESS J1745-290 originate from the same production mechanism, any emission model should at least meet the following requirements: (i) a power-law spectrum from $100 \mathrm{MeV}$ to $20 \mathrm{TeV}$ with an energy cut-off at $\sim 10 \mathrm{TeV}$, (ii) no hint for variability on timescale from minutes to years, (iii) the emission region of the $\mathrm{TeV}$ source is point-like and coincident with the position of $\mathrm{Sgr} \mathrm{A}^{*}$, and its intrinsic size is less than 1'; and (iv) a possible small extension of the GeV source. 


\section{Diffusion emission at $\mathrm{TeV}$ energies}

Follow-up VHE observations provided a more detailed view of the overall Central Molecular Zone (CMZ) and led to the detection of a diffuse emission in the central $300 \mathrm{pc}$ of the GC [4] extended along the Galactic plane. The spatial correlation of the $\mathrm{TeV}$ emission with the giant molecular clouds of the CMZ first hinted for acceleration of hadronic CRs in this region [4], where the $\gamma$-rays result from decays of $\pi_{0}$ produced by the interactions of relativistic protons with the ambient gas.

The large photon statistics accumulated over 10 year observations together with improvements in the data analysis method, provide now a refined picture of the spectral and spatial properties of the diffuse emission of the CMZ [5]. As shown in Fig. 1, the sky map in VHE $\gamma$-rays shows a strong, although not linear, correlation between the brightness distribution of $\gamma$-rays and the locations of massive gas-rich complexes. The $\gamma$-ray morphology of the CMZ is both determined by the location and the particle injection rate history of the $\mathrm{CR}$ accelerator(s) responsible for the relativistic protons, and by the gas density distribution. The spatial morphology seen now in VHE $\gamma$-rays is a unique proxy to probe the CR distribution within the CMZ.

If the $\gamma$-ray emission is completely due to the decay of $\pi_{0}$ produced in $p p$ interactions, the $\gamma$-ray luminosity $L_{\gamma}$ above energy $E$ is related to the total energy of CR protons $W_{p}$ by $L_{\gamma}(>E) \simeq \eta_{\mathrm{N}} W_{p}(>$ $10 E) / t_{p p \rightarrow \pi_{0}}$, where $t_{p p \rightarrow \pi_{0}}=1.6 \times 10^{8} \mathrm{yr}\left(1 \mathrm{~cm}^{-3} / n_{\mathrm{H}}\right)$ is the proton energy loss timescale due to $\pi_{0}$ production in an environment of hydrogen gas of density $n_{\mathrm{H}}$, and $\eta_{\mathrm{N}} \simeq 1.5$ accounts for the presence of nuclei heavier than hydrogen in both CRs and interstellar matter. With the measurements of the
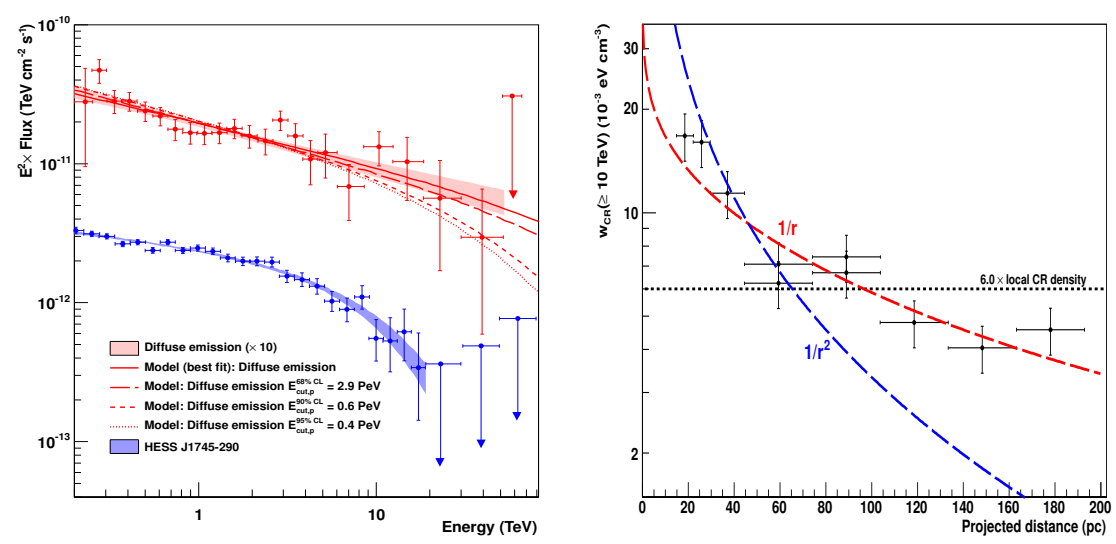

Figure 2. Left panel: VHE $\gamma$-ray spectra of the diffuse emission and HESS J1745-290. Arrows represent $2 \sigma$ flux upper limits. The $1 \sigma$ confidence bands of the best-fit spectra of the diffuse and HESS J1745-290 are shown in red and blue shaded areas, respectively. The red lines show the numerical computations assuming that $\gamma$-rays result from the decay of neutral pions produced by $p p$ interactions. Right panel: Spatial distribution of the CR energy density versus projected distance from Sgr A*. The vertical and horizontal error bars show the $1 \sigma$ statistical plus systematic errors and the bin size, respectively. Fits to the data of a $1 / r$ (red line), a $1 / r^{2}$ (blue line) and a homogeneous (black line) CR density radial profile integrated along the line of sight are shown. The best fit of a $1 / \mathrm{r}^{\alpha}$ profile to the data is found for $\alpha=1.10 \pm 0.12(1 \sigma)$.

target masses which can be inferred using tracer molecules such as $\mathrm{CS},{ }^{12} \mathrm{C}^{16} \mathrm{O}$ and $\mathrm{HCN}$ [5], the CR energy density $w_{\mathrm{CR}}$ averaged along the line of sight, can be obtained. The right panel of Fig. 2 shows the radial profile of $w_{\mathrm{CR}}$ for $\mathrm{E}>10 \mathrm{TeV}$ CRs up to a radius of $200 \mathrm{pc}$ for a GC distance of $8.5 \mathrm{kpc}$, 
determined from the $\gamma$-ray luminosity and the amount of target gas. This high energy density in the $\mathrm{CMZ}$ is found to be an order of magnitude larger than that of the CR "sea" that universally fills the Galaxy, while the energy density of low energy $(\mathrm{GeV}) \mathrm{CRs}$ in this region has a level comparable to it [5]. This requires the presence of one or more accelerators of multi-TeV particles operating in the CMZ. The measurement of the CR density profile clearly supports the $1 / r$ dependence over the entire CMZ. The $1 / r^{2}$ and constant profiles, the former being expected if CRs are advected in a wind, and the latter in the case of a single burst-like event of CR injection, are significantly disfavored.

The CR radial profile points towards an accelerator located in the inner $10 \mathrm{pc}$ of the GC. The $1 / r$ behavior of the CR density up to $200 \mathrm{pc}$ indicates a quasi-continuous injection of protons that diffuse into the CMZ from a centrally-located accelerator within the $10 \mathrm{pc}$ of Sgr A*. The average injection rate of particles is found to be $Q_{\mathrm{p}}(>10 \mathrm{TeV}) \simeq 4 \times 10^{37}\left(D / 10^{30} \mathrm{~cm}^{2} \mathrm{~s}^{-1}\right) \mathrm{erg} \mathrm{s}^{-1}$. The diffusion coefficient $D$ depends on the power spectrum of the turbulent magnetic field, which is unknown in the CMZ region. Yet, the diffusive nature of the propagation is constrained by the condition $R^{2} / 6 D \ll R / c$. For a radius $R$ of the CMZ region of $200 \mathrm{pc}$, this implies $Q_{\mathrm{p}} \ll 1.2 \times 10^{38} \mathrm{erg} \mathrm{s}^{-1}$. The supermassive black hole $\mathrm{Sgr} \mathrm{A}^{*}$ at the GC is the most plausible supplier of ultra-relativistic protons and nuclei where these particles could have been accelerated either in the accretion flow (i.e. in the immediate vicinity of the black hole) or somewhat further away - for example, at the site of termination of an outflow [5].

\section{The discovery of a Pevatron}

Given the available statistics, the energy spectrum of the diffuse VHE $\gamma$-ray emission has been extracted from an annulus centred at Sgr A* (see right panel of Fig. 1). The best fit to the data is found for a spectrum following a power law extending with a photon index of $\sim 2.3$ to energies up to tens of $\mathrm{TeV}$ without an energy cut-off or break, as shown in the left panel of Fig. 2. This is the first time that such a $\gamma$-ray spectrum, arising from hadronic interactions, has been detected. Since these $\gamma$-rays result from decays of $\pi_{0}$ produced by $p p$ interactions, the derivation of such a hard power-law spectrum implies that the spectrum of the parent protons should extend to energies close to $1 \mathrm{PeV}$. Assuming a cut-off in the parent proton spectrum, the corresponding secondary $\gamma$-ray spectrum deviates from the H.E.S.S. data at $68 \%, 90 \%$ and $95 \%$ confidence levels for cut-offs at $2.9 \mathrm{PeV}, 0.6 \mathrm{PeV}$ and $0.4 \mathrm{PeV}$, respectively. This establishes the first robust detection of a VHE cosmic hadronic accelerator which operates as a source of $\mathrm{PeV}$ particles, i.e. a Pevatron.

If Sgr A* is indeed the particles' source, the required acceleration rate would exceed by two or three orders of magnitude its current bolometric luminosity, and would constitute at least $1 \%$ of the current power produced by accretion onto the supermassive black hole. Given the relativelymodest current accretion rate of Sgr $\mathrm{A}^{*}$ and that at certain epochs this supermassive black hole could have operated at a much higher accretion rate, this higher rate could also have facilitated greater CR production rates. An average acceleration rate of $10^{39} \mathrm{erg} \mathrm{s}^{-1}$ of $\mathrm{E}>10 \mathrm{TeV}$ protons over the last $10^{6}$ $10^{7}$ years would be sufficient to explain the flux of CRs around the "knee", an energy spectrum feature around $1 \mathrm{PeV}$. If so, $\mathrm{Sgr} \mathrm{A}^{*}$ should be considered as a viable alternative to supernova remnants as a source of PeV Galactic cosmic rays.

\section{References}

[1] F. Aharonian et al. (H.E.S.S. Collaboration), Astron. Astrophys. 425, L13 (2004)

[2] F. Aharonian et al. (H.E.S.S. Collaboration), Astron. Astrophys. 503, 817 (2009)

[3] F. Acero et al. (H.E.S.S. Collaboration), Mon. Not. Roy. Ast. Soc. 402, 1877 (2010)

[4] F. Aharonian et al. (H.E.S.S. Collaboration), Nature 439, 695 (2006)

[5] A. Abramowski et al. (H.E.S.S. Collaboration), Nature 531, 476 (2016) 